\begin{tabular}{|c|l|}
\hline Title & Electron swarm parameters in water vapour \\
\hline Author(s) & Hasegawa, H.; Date, H.; Shimozuma, M. \\
\hline Citation & Journal of Physics D A pplied Physics, 40(8), 2495-2498 \\
\hline https://doi.org/40.1088/0022-3727/40/8/012 \\
\hline Issue Date & 2007-04.21 \\
\hline Doc URL & http://hdl.handle.net/2115/26412 \\
\hline Rights & Copyright $\odot$ 2007 IOP Publishing Ltd. \\
\hline Type & article (author version) \\
\hline File Information & JPD40-8.pdf \\
\hline
\end{tabular}

Instructions for use 


\title{
Electron Swarm Parameters in Water Vapor
}

\author{
H. Hasegawa ${ }^{1}$, H. Date ${ }^{2 *}$ and M. Shimozuma ${ }^{3}$ \\ ${ }^{1}$ Tomakomai National College of Technology, Tomakomai 059-1275, Japan \\ ${ }^{2}$ School of Medicine, Hokkaido University, Sapporo 060-0812, Japan \\ ${ }^{3}$ Hokkaido Institute of Technology, Sapporo 006-8585, Japan
}

\begin{abstract}
Electron swarm parameters, such as the drift velocity and the ionization coefficient, in water vapor have been measured for relatively wide ranges in reduced electric fields $(\mathrm{E} / \mathrm{N})$ at room temperature. The drift velocity $\left(\mathrm{W}_{\mathrm{m}}\right)$ was obtained based upon the arrival-time spectra of electrons by using a double-shutter drift tube for the $\mathrm{E} / \mathrm{N}$ from 60 to $1000 \mathrm{Td}$, while the first and second ionization coefficients ( $\alpha$ and $\gamma$ ) were determined by the steady-state Townsend (SST) method from 50 to $3000 \mathrm{Td}$. The comparison between the results and other data in the literature shows that our results for both the drift velocity and the effective ionization coefficient are lower than those of the other data in the above ranges.
\end{abstract}

PACS numbers: $\quad$ 51.50.+v, 52.80.-s, 52.20.Fs, 34.80.-i

*Corresponding author: H. Date

Postal address: N12-W5, Kita-ku, Sapporo 060-0812, JAPAN

Email address: date@cme.hokudai.ac.jp

Tel: +81-11-706-3423, Fax: +81-11-706-4916 


\section{Introduction}

Water vapor and mist are important components governing the air discharge and the surface discharge on insulators, in which the electrical conductivity leading to a current path is changed by a small amount of water (e.g., Lowke 1992, Falkenstein and Coogan 1997). The water molecule has many excitation modes and ionization channels for absorbing energy by electron collision processes. The collision processes of electrons are essential not only in the gas discharges (or plasmas) but also in the effects of radiation damage of bio-tissues. When $\mathrm{X}$-rays or $\gamma$-rays are incident on water, they produce photoelectrons and Compton electrons as principal interactions. Thus, the energy of the incident high-energy photons is transferred to water molecules through the collisions by abundant electrons as the final physical stage (Huels et al. 2003).

To investigate the collision processes by electron impact, the electron swarm experiment is an effective and reliable method to observe a macroscopic behavior of many electrons in a manner comprising the hydrodynamic equilibrium of energy gain and loss processes. In the hydrodynamic equilibrium condition, the energy distribution of electrons can be considered unique as a function of the reduced electric field $\mathrm{E} / \mathrm{N}$, and the swarm parameters such as drift velocity and ionization coefficient are also constant (Date et al. 1998). This means that one can connect the microscopic collision probabilities, i.e. cross sections, uniquely to the swarm parameters as integrated statistical quantities.

This paper reports the drift velocity $\mathrm{W}_{\mathrm{m}}$ defined by the arrival-time spectra (ATS) (Kondo and Tagashira 1990) and the ionization coefficient of electrons by the steady-state Townsend (SST) (Hasegawa et al. 1985) method in water vapor for a relatively wide E/ $\mathrm{N}$ region. The drift velocity was measured at E/N from 60 to $1000 \mathrm{Td}$, while the first and second ionization coefficients were determined from 50 to $3000 \mathrm{Td}$. The results should be of use to estimate the cross sections of electron collision with water molecule for energy below about $100 \mathrm{eV}$ by means of the Boltzmann equation analysis and the Monte Carlo simulation method, and the 
dielectric breakdown voltage of gases including water.

\section{Experimental methods}

\subsection{Arrival-time spectra method}

The measurement of the drift velocity was performed based on the arrival-time spectra (ATS) method (Kondo and Tagashira 1990, Date et al. 1990) for evaluating the drift velocity $\mathrm{W}_{\mathrm{m}}$. Here, $\mathrm{W}_{\mathrm{m}}$ is defined from the distribution of ATS of electrons as a coefficient of the transport equation that is given by the interchange of space and time in the conventional continuity equation (Kondo and Tagashira 1990) as follows.

$$
\frac{\partial \mathrm{n}}{\partial \mathrm{z}}=\alpha^{(0)} \mathrm{n}-\alpha^{(1)} \frac{\partial \mathrm{n}}{\partial \mathrm{t}}+\alpha^{(2)} \frac{\partial^{2} \mathrm{n}}{\partial \mathrm{t}^{2}}-\alpha^{(3)} \frac{\partial^{3} \mathrm{n}}{\partial \mathrm{t}^{3}}+\cdots
$$

where

$$
\begin{aligned}
& \alpha^{(0)}=\frac{1}{\mathrm{~N}(\mathrm{z})} \frac{\mathrm{dN}(\mathrm{z})}{\mathrm{dz}}=\bar{\alpha} \\
& \alpha^{(1)}=\frac{\mathrm{d}\langle\mathrm{t}\rangle}{\mathrm{dz}}=\frac{1}{\mathrm{~W}_{\mathrm{m}}} \\
& \mathrm{N}(\mathrm{z})=\int_{0}^{\infty} \mathrm{n}(\mathrm{z}, \mathrm{t}) \mathrm{dt} .
\end{aligned}
$$

Here, $\mathrm{n}(\mathrm{z}, \mathrm{t})$ is the electron number density at a position $\mathrm{z}$ and time $\mathrm{t}, \bar{\alpha}$ is the effective ionization coefficient, and $<\mathrm{t}>$ represents the average of the arrival time of electrons at every location. The coefficients in the right-hand side of equation (1) are the parameters describing the behavior of the ATS at the spatial location of the electrode capturing arrival electrons. The values $\mathrm{W}_{\mathrm{m}}$ are determined by the derivative of the mean arrival time $<\mathrm{t}>$ with respect to the drift distance as in equation (4).

The experimental apparatus and procedure for the ATS method are the same as those described by Hasegawa et al (1996).

\subsection{Steady-state Townsend method}


The effective ionization coefficient $\bar{\alpha}$ was determined by the steady-state Townsend (SST) method. We have used the SST method for obtaining $\bar{\alpha}$ because it enables us to make it for a wider range of E/Ns than that by the ATS method owing to our apparatus limitation stated in subsection 2.3. The experimental apparatus and procedure are similar to those by Hasegawa et al (1985). In the experiment, the Townsend first ionization coefficient $\alpha$ and the electron attachment coefficient $\eta$ are determined by fitting the Townsend's theoretical equation to the experimental current growth curve.

$$
\mathrm{I}=\mathrm{I}_{0} \frac{\frac{\alpha}{\alpha-\eta} \exp \{(\alpha-\eta) \mathrm{d}\}-\frac{\eta}{\alpha-\eta}}{1-\gamma_{\mathrm{T}} \frac{\alpha}{\alpha-\eta}[\exp \{(\alpha-\eta) \mathrm{d}\}-1]}
$$

Equation (5) is the Townsend's equation for the SST experiment where $\mathrm{I}_{0}$ is the initial current value and $\gamma_{\mathrm{T}}$ is the secondary coefficient. If the secondary effect appears significantly, we can consider that the attachment process is negligible (i.e., $\eta \cong 0$ ). Thus, equation (5) can be transformed to

$$
I=I_{0} \frac{\exp (\alpha d)}{1-\gamma_{T}\{\exp (\alpha d)-1\}} .
$$

Contrary to this, if the attachment occurs considerably, the secondary coefficient would be nearly zero. Then we have

$$
I=I_{0}\left[\frac{\alpha}{\alpha-\eta} \exp \{(\alpha-\eta) d\}-\frac{\eta}{\alpha-\eta}\right]
$$

The fitting each equation to both cases of the experimental condition is performed using the linearized least-square-method, and the effective ionization coefficient $\bar{\alpha}$ is deduced as $\alpha-\eta$.

\subsection{Purity of water vapor and procedure of infusion}

Water sample for the present experiment was produced by the ionic exchange EDI module (WEX3, YAMATO SCIENTIFIC CO., LTD). Specifications of the module are: purity $>3$ $\mathrm{M} \Omega \cdot \mathrm{cm}$, conductivity $<1000 \mu \mathrm{S} / \mathrm{cm}$, hardness $<500 \mathrm{ppm}$, dissolved carbon dioxide $<30 \mathrm{ppm}$, 
Fouling Index $(\mathrm{FI})<12$, residual chlorine $<3 \mathrm{ppm}$. The sample water is infused into the vacuum chamber (for the ATS or SST experiment) from a conical flask reservoir through a stainless mount. The water vaporizes immediately just after opening the infusion valve because the vacuum chamber is highly evacuated (below $10^{-7}$ Torr) beforehand.

In the ATS apparatus, a multiple guard-ring is equipped to make uniform electric fields for the discharge space. Contrary to the inside of the guard-ring, a lot of non-uniform electric fields exist outside the guard-ring in several locations. Therefore, we were unable to apply the voltage to the discharge electrodes higher than that in the case of SST experiment (using the Harrison type electrodes). In order to obtain the same order of E/ $\mathrm{N}$ values, we had to lessen the pressure in the ATS experiment to 0.1-1.0 Torr at the $0^{\circ} \mathrm{C}$-reduced temperature, as opposed to 0.5-5.5 Torr in the SST experiment. The voltage limit of our ATS apparatus is $350 \mathrm{~V}$ for nitrogen gas in 1.0 Torr pressure.

\section{Results and discussion}

Figure 1 shows the drift velocity $\mathrm{W}_{\mathrm{m}}$ compared with other data in the literature. The present $\mathrm{W}_{\mathrm{m}}$ data are 20\% (at maximum) lower than the drift velocity data by Ness and Robson (1988) in $\mathrm{E} / \mathrm{N}=200-300 \mathrm{Td}$. At $\mathrm{E} / \mathrm{N}$ around $100 \mathrm{Td}$, the result is $20-25 \%$ lower than that of Yousfi and Benabdessadok (1996) and of Ryzko (1966), while the results are 9\% (at maximum) larger those that of Wilson et al (1975) below $\mathrm{E} / \mathrm{N}=80 \mathrm{Td}$. The discrepancy here may be attributable to the difference in the definition of the drift velocity. We were unable to identify the precise definition of the drift velocity in the literature, particularly experimental values. However, the difference arising from the definition is supposed to occur in a highly ionizing condition under very high E/Ns; and so, it is presumably caused by the difference of vaporized water impurity including ions and minute contaminants rather than the difference of the definition. Unfortunately, the vaporizing procedure and impurity of water were also unstated in detail in the literature. 
The effective first ionization coefficient (per gas density $\mathrm{N}$ ) is shown in Fig.2. The values of the coefficient are also lower than the other reported data, particularly in a high E/N region above $800 \mathrm{Td}$ and a lower region below $200 \mathrm{Td}$. The differences in both the regions are more than $50 \%$. This difference may also be attributable to the minute difference of gas sample purity. The effects of impurity of gas sample on the swarm parameters have been known in inert gases. Dutton et al (1983) presented that a difference of the gas purity may cause a large difference of the ionization coefficient up to 2-3 times of magnitude. As for the impurity elements of water vapor, some molecules such as carbon dioxides, organic materials and metals (potassium and calcium, etc.) are presumed. These are reactive to low energy electrons below a few tens of eV, and may have been dissolved in the water sample before vaporization. Since the ionization threshold and electron attachment cross sections of water molecule lie in the region of energy between 4 and $15 \mathrm{eV}$ (e.g. Itikawa and Mason, 2005), the effective ionization coefficient must be very sensitive to a small amount of difference in the gas components. For example, the carbon dioxide molecule has large vibrational excitation cross sections in the energy from 0.1 to $40 \mathrm{eV}$ (e.g. Hasegawa et al, 1998). For the discrepancy in the high $\mathrm{E} / \mathrm{N}$ region above $800 \mathrm{Td}$, we may not rule out the possibility that the electron swarm is not in the hydrodynamic condition under such a high E/N. However, we were not able to recognize any symptom of instabilities arising from the space charge field in the ionization growth curves. In our measurement apparatus, the gap length of electrodes is 2-6.5 mm, and the gas pressure $\mathrm{p}$ is 0.5 Torr for $\mathrm{E} / \mathrm{N}=3000 \mathrm{Td}$. For the low $\mathrm{E} / \mathrm{N}$ region, the coefficient is specifically plotted in Fig.3. From the curve in Fig.3, the limiting $E / N$ (for $\alpha-\eta=0$ ) is found to be about $130 \mathrm{Td}$. In the E/N region around $100 \mathrm{Td}$, the effective ionization coefficient is very sensitive to the ionization and attachment processes, and a statistical nature may appear significantly.

Figure 4 shows the total secondary ionization coefficient $\gamma_{\mathrm{T}}$. Every point plotted corresponds to one measurement. To our knowledge, the secondary coefficient for water 
vapor has scarcely been reported. As in Fig.4, the total effects on the electron emission from the cathode by positive ions and photons are found to be from 0.0001 to 0.03 for $\mathrm{E} / \mathrm{N}$ from 500 to 3000 Td.

\section{Conclusion}

In this paper, the drift velocity $\left(\mathrm{W}_{\mathrm{m}}\right.$ ) was obtained by using a double-shutter drift tube for the $\mathrm{E} / \mathrm{N}$ from 60 to $1000 \mathrm{Td}$, and the first and second ionization coefficients ( $\alpha$ and $\gamma$ ) were determined by the steady-state Townsend (SST) method from 50 to $3000 \mathrm{Td}$. In general, our results for both the drift velocity and the effective ionization coefficient $(\alpha-\eta)$ are lower than those reported in the literature. We thought that the discrepancy could be attributable mainly to the impurity of water vapor sample. However, the problem related with the impurity needs to be investigated with further examinations. The limiting E/ $\mathrm{N}$ value was about $130 \mathrm{Td}$ in the present experiment, and the secondary ionization coefficient ranged from 0.0001 to 0.03 for E/N from 500 to 3000 Td with a monotonic increase. To our knowledge, the secondary coefficient for water vapor was reported for the first time.

\section{Acknowledgment}

The authors are most grateful to Professors H Tagashira and K Yoshida for helpful discussions and useful suggestions. This work was financially supported in part by the Japan Science and Technology Agency. 


\section{References}

Date H, Kondo K and Tagashira H 1990 J. Phys. D: Appl. Phys. 23 1384-9

Date H, Ventzek P L G, Kondo K, Hasegawa H, Shimozuma M and Tagashira H $1998 \mathrm{~J}$. Appl. Phys. 83(8), 4024-9

Dutton J, Mat Yunus W M and Williams A W 1983 Proc. $16^{\text {th }}$ ICPIG (Dusseldorg) (Henkel) 2 158-9

Falkenstein Z and Cooran J J 1997 J. Phys. D: Appl. Phys. 30 817-25

Hasegawa H, Sato Y, Murai K, Shimozuma M and Tagashira H 1985 J. Phys. D: Appl. Phys. $181361-70$

Hasegawa H, Date H, Shimozuma M, Yoshida K and Tagashira H 1996 J. Phys. D: Appl. Phys. 29 2664-7

Huels M A, Boudaïffa B, Cloutier P, Hunting D and Sanche L 2003 J. Am. Chem. Soc. 125, 4467-77

Itikawa Y and Mason N 2005 J. Phys. Chem. Ref. Data 34(1), 1-22

Kondo K and Tagashira H 1990 J. Phys. D: Appl. Phys. 23 1175-83

Lowke J J 1992 J. Phys. D: Appl. Phys. 25, 202-10

Lowke J J and Ress J A 1963 Aust. J. Phys. 16, 447- 53

Ness K F and Robson R E 1988 Phys. Rev. A 38(3), 1446-56

Pack J L, Voshall R E and Phelps A V 1962 Phys. Rev. 127, 6, 2084-9

Prasad A N and Craggs J D 1960 Proc. Phys. Soc. London 76, 223-32

Risbud A V and Naidu M S 1979 J. Phys. (France) Colloq. 40, 77

Ryzko H 1966 Arkiv. Fysik. 32,1-18

Wilson J L, Davis F J, Nelson D R and Compton R N 1975 J. Chem. Phys. 62(10), 4204-12 Yousfi M and Benabdessadok M. D 1996 J. Appl. Phys. 80(12), 6619-30 


\section{Figure captions}

\section{Figure 1.}

Comparison of the drift velocity. The point in the present work represents the mean arrival-time drift velocity $\mathrm{W}_{\mathrm{m}}$.

\section{Figure 2.}

Comparison of the effective first ionization coefficient, $\bar{\alpha}=\alpha-\eta$.

\section{Figure 3.}

The effective first ionization coefficient in a low $\mathrm{E} / \mathrm{N}$ region.

\section{Figure 4.}

The secondary ionization coefficient as a function of E/N. Each point plotted corresponds to one measurement. 


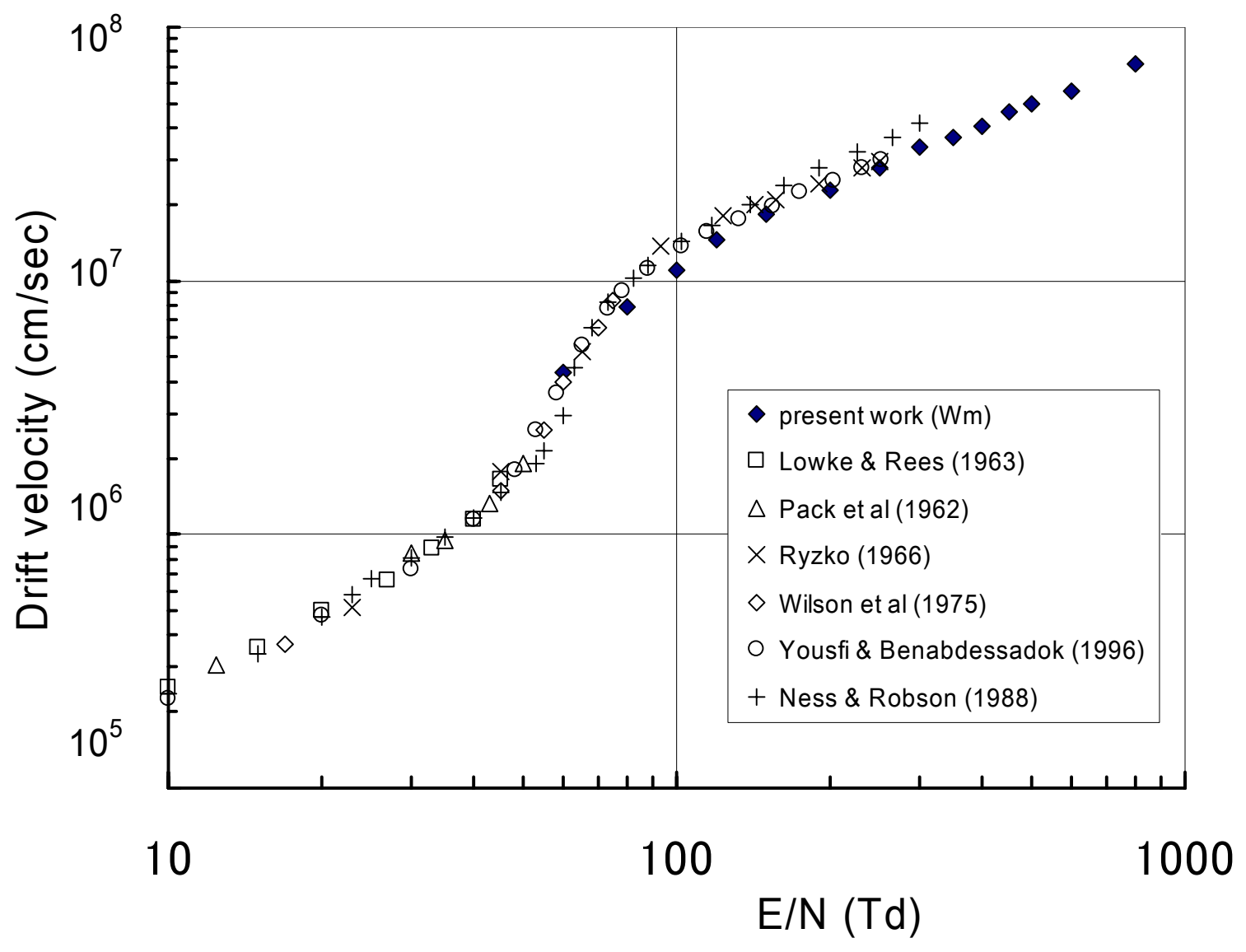

Figure 1 .

$H$. Hasegawa et al. 


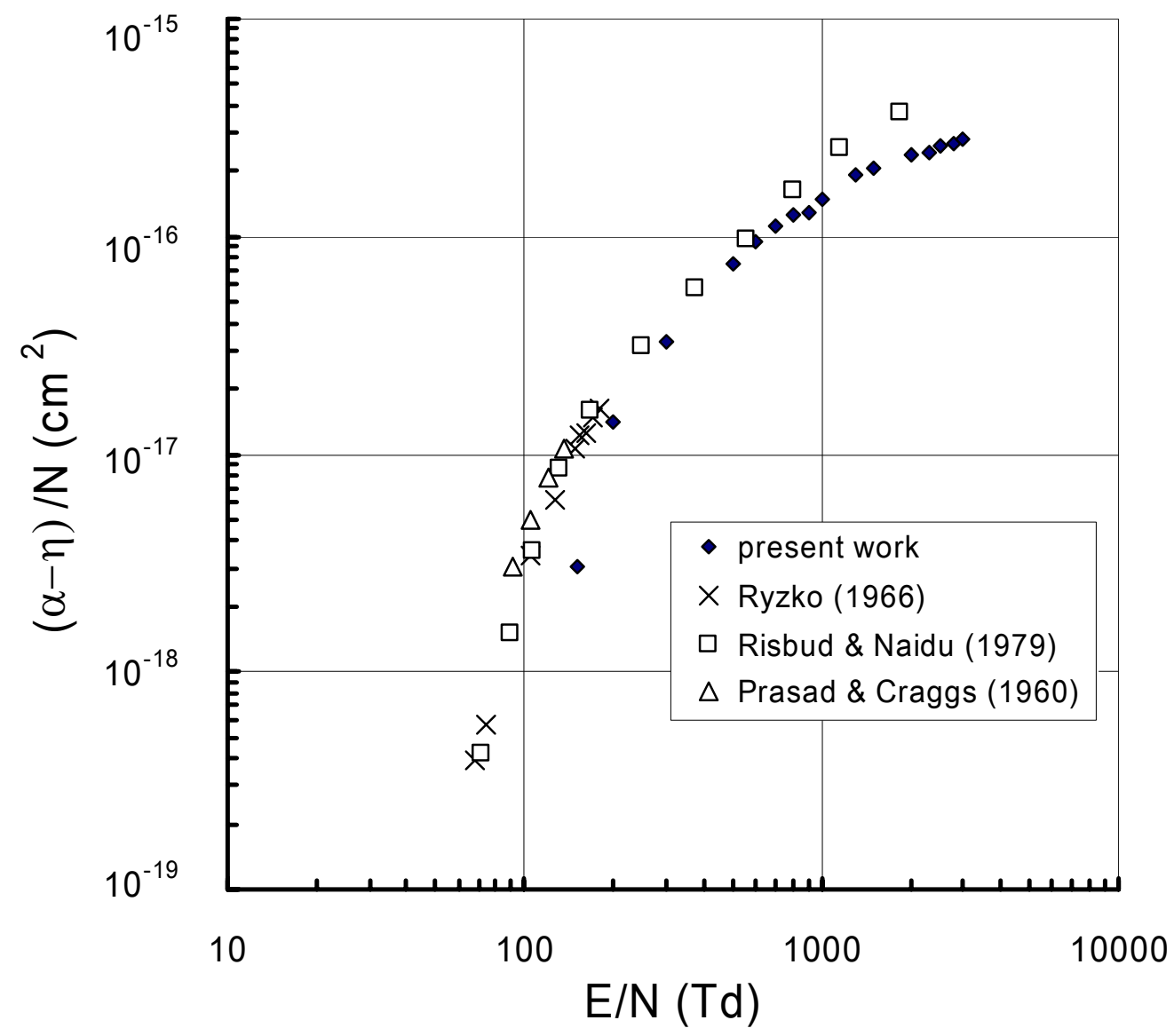

Figure 2.

H. Hasegawa et al. 


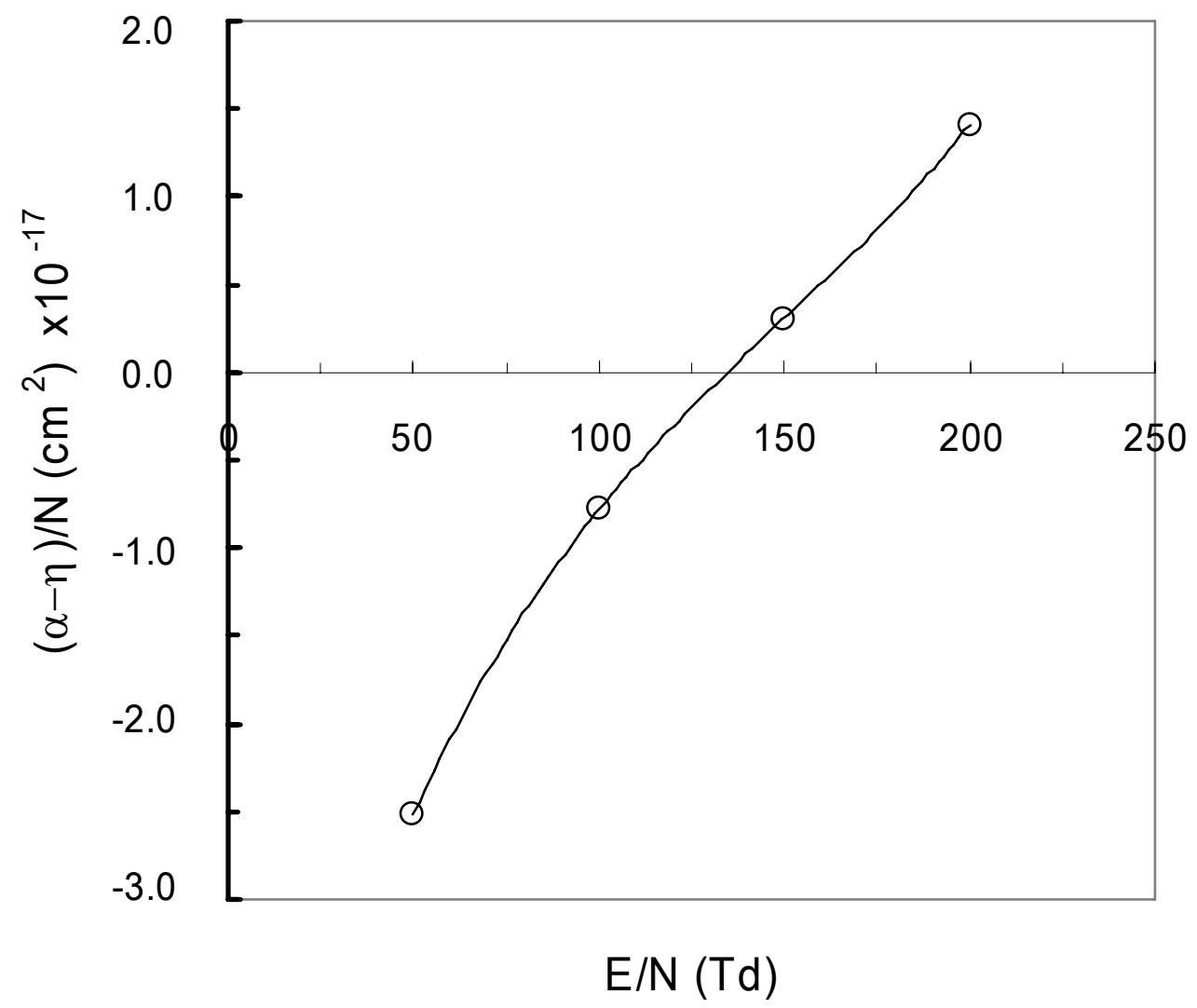

Figure 3.

H. Hasegawa et al. 


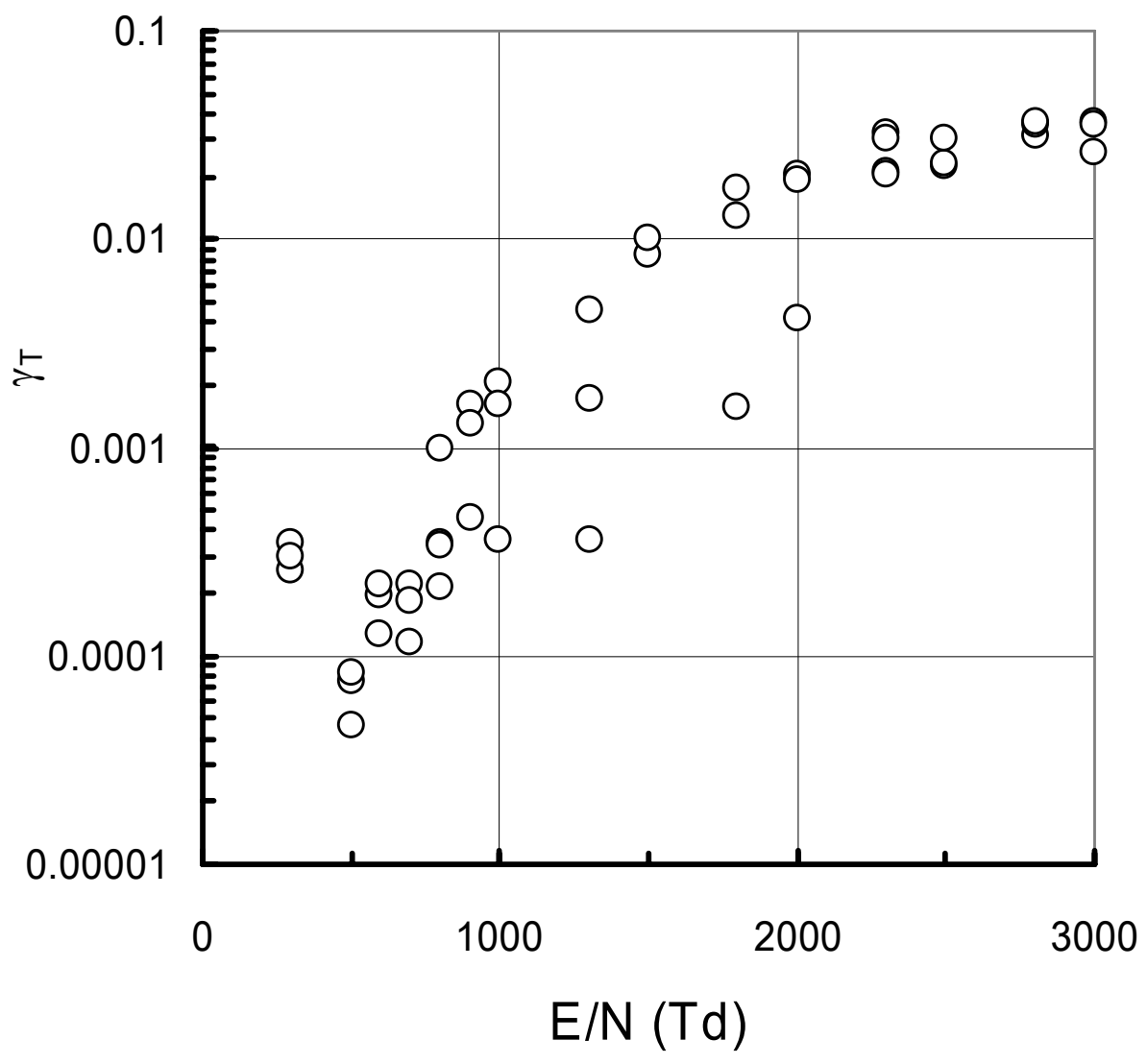

Figure 4.

H. Hasegawa et al. 